\title{
ASO Visual Abstract: Characteristics and Outcomes in Pediatric Non-Central Nervous System Malignant Rhabdoid Tumors-A Report from the National Cancer Database
}

\author{
Katrina M. Morgan, MD ${ }^{1}$ (i), Vei Shaun Siow, $\mathrm{MD}^{1}$, Stephen Strotmeyer, $\mathrm{PhD}^{2}$, Kenneth W. Gow, $\mathrm{MD}^{3}$, and \\ Marcus M. Malek, MD $^{2}$ \\ ${ }^{1}$ University of Pittsburgh Medical Center, F677 Presbyterian Hospital, Pittsburgh, PA ; ${ }^{2}$ UPMC Children's Hospital of \\ Pittsburgh, Pittsburgh, PA; ${ }^{3}$ Seattle Children's Hospital, Seattle, WA
}

Pediatric non-central nervous system malignant rhabdoid tumor is a rare and aggressive malignancy. The National Cancer Database is the largest and most contemporary dataset of this cohort, and verified that infants and those with metastatic disease had worse survival outcomes (https://doi.org/10. 1245/s10434-021-10370-x).

DISCLOSURE The authors declare that they have no competing interests

Characteristics and Outcomes in Pediatric Non-Central
Nervous System Malignant Rhabdoid Tumor

(C) Society of Surgical Oncology 2021

Published Online: 22 July 2021

K. M. Morgan, MD

e-mail: morgankm2@upmc.edu; kmmorgan123@gmail.com
Publisher's Note Springer Nature remains neutral with regard to jurisdictional claims in published maps and institutional affiliations. 